\title{
RECURSOS EDUCATIVOS TIC EN LA ENSEÑANZA MUSICAL PIANÍSTICA
}

\author{
Concepción de Castro \\ Conservatorio Profesional de Música de León - Universidad Nacional de Educación a Distancia \\ ccastropu@educa.jcyl.es
}

Este artículo aborda el análisis de los recursos educativos TIC más utilizados en los procesos de enseñanza-aprendizaje de la práctica musical pianística. Los recursos tecnológicos y las grabaciones de audio y vídeo con fines pedagógicos constituyen uno de los instrumentos más valorados en los contextos de enseñanza musical especializada. A partir de una experiencia didáctica basada en la observación, descripción y detección de estilos de aprendizaje de un grupo de estudiantes de conservatorio de la especialidad de piano, se plantea una propuesta de práctica que utiliza la grabación didáctica como un recurso educativo versátil en el aprendizaje musical.

Descriptores: TIC, tecnología educativa, recursos educativos, estilos de aprendizaje, aprendizaje musical, didáctica pianística

This article discusses the analysis of the ICT educational resources that are most commonly used in the piano teaching and learning process. Technological resources and audio and video recordings for educational purposes are one of the most valuable tools for specialised musical education. After analysing an educational experience based on observation, description and identification of learning styles with a group of conservatory piano students, a practical teaching approach that uses recordings as a versatile educational resource in musical learning is proposed.

Keywords: ICT, educational technology, educational resources, learning styles, musical learning, piano teaching

\section{INTRODUCCIÓN}

Los usos de las tecnologías de la información y la comunicación (TIC) en la sociedad actual se encuentran integrados en todos los aspectos de la actividad humana, generando nuevas formas de comunicación y conducta, que afectan a la manera en la que los seres humanos aprenden y se relacionan con su entorno. El hecho musical constituye uno de los fenómenos más característicos de esa actividad humana, en cualquiera de sus culturas. Las actividades musicales y los mecanismos que operan en ellas, movilizan en las personas que las ejecutan una serie de habilidades, competencias y destrezas altamente complejas, dirigidas a acciones vitales propias del ser humano como la comunicación y las emociones. Estos procesos, interacciones, relaciones y sus efectos han suscitado y lo siguen haciendo, un gran interés en varios campos del conocimiento. Esto unido al progreso tecnológico, ha propiciado que en los entornos educativos hayan surgido nuevos paradigmas y espacios de aprendizaje, así como un replanteamiento de las posturas pedagógicas asumidas en la cultura occidental.

En las líneas siguientes se expone una valoración del uso de las TIC en la enseñanza musical especializada, con especial atención al uso de las vídeo-grabaciones, como recurso con el cual examinar las múltiples dimensiones del acto musical (sea éste creativo, ejecutivo, interpretativo o de aprendizaje); potenciar el desarrollo de habilidades musicales concretas (auditivas, visuales, motrices) y obtener información de distintas situaciones de aprendizaje. Para ello, será necesario antes abordar cuestiones como las implicaciones educativas que la tecnología tiene en el aprendizaje musical, las aportaciones de ésta desde los diferentes estilos de aprendizaje y el planteamiento de una situación de aprendizaje concreta.

Los nuevos entornos de aprendizaje hacen que la actividad docente se extienda más allá de lo que acontece en el aula o en las instituciones educativas, lo cual obliga al profesorado a asumir nuevas tareas y funciones, necesarias para que las actuaciones pedagógicas tengan una dimensión educativa 
plena, no sólo de mera instrucción, en la que se preserve lo mejor de nuestro legado cultural y pedagógico, al servicio del desarrollo integral de la persona ${ }^{1}$.

\title{
HERRAMIENTAS TIC EN LA ENSEÑANZA MUSICAL INSTRUMENTAL
}

Los entornos de aprendizaje musical, al igual que otros espacios educativos, se han visto afectados por las transformaciones derivadas de la progresiva implementación de la tecnología, generando una serie de consecuencias positivas, como la creación de nuevos espacios, formas y procedimientos de aprendizaje; y otras negativas, como la emergencia de ambientes sobre-estimulantes que dificultan las tareas de atención y concentración o la manipulación de gustos, usos y actitudes, derivados de una exposición no controlada a determinados tipos de contenidos.

Los centros de educación musical han tratado de adaptarse a los nuevos tiempos, incorporando gradual y progresivamente a sus proyectos educativos y curriculares nuevas estrategias y recursos tecnológicos dirigidos a favorecer las experiencias de aprendizaje, facilitar la comunicación entre los diferentes miembros de la comunidad educativa y difundir sus actividades formativas y musicales. Entre los recursos educativos TIC más utilizados en las prácticas de enseñanza aprendizaje de estos centros encontramos:

- Grabaciones digitales de audio y vídeo: Este recurso es uno de los que mayor evolución ha experimentado en los últimos años. De las primeras grabadoras y mini-disc, cuyos archivos requerían cierta complejidad para su manejo, se ha pasado a modernos dispositivos, cuyos archivos se editan, transfieren y difunden de manera, casi intuitiva, por cualquier usuario (los programas y dispositivos asociados a estos recursos han simplificado su uso). Las grabaciones son recursos de gran utilidad para desarrollar una audición crítica y en el caso del vídeo, para observar detalles y aspectos de la ejecución que pasan inadvertidos a lo largo de su realización. El gran pianista Alfred Brendel (2013) expresa así la valoración de este recurso en relación a la práctica de estudio en De la A a la Z de un pianista:

\begin{abstract}
Los requisitos son disponer de un buen instrumento; una buena edición del texto original; una técnica interpretativa que no ocasiones ningún daño físico al pianista; trabajo concentrado, pero no encarnizado; ambición, paciencia; la elección de las piezas adecuadas; y en último lugar, pero no por ello menos importante, la facultad de escucharse a sí mismo durante la ejecución. Esta facultad habrá que ir desarrollándose en el transcurso del tiempo y con ayuda de una grabadora. Cuando cumplí veinte años, unas personas muy amables me regalaron un magnetófono Revos, todavía lo conservo (Brendel, 2013, p. 102).
\end{abstract}

- Recursos audio digitales y software musical “Minus One”: Dentro de esta categoría agrupamos aquellos recursos audiovisuales e informáticos que permiten la práctica musical de uno o varios ejecutantes, en diferentes grados de interacción con la reproducción simultánea de una gran variedad de elementos sonoros: acompañamientos didácticos y/o específicos, partes en agrupaciones camerísticas u orquestales, etc.: http://musicminusone.com.

- Editores de partituras: Al igual que los procesadores de texto u otras herramientas ofimáticas los editores de partituras han desarrollado una múltiple funcionalidad, que ha aumentado su potencialidad, tanto en calidad sonora, como visual y educativa, de manera que tareas como la digitalización de partituras que en un principio podían ser tediosas, actualmente se desempeñan con fluidez y facilidad. Entre los programas más conocidos se encuentra Sibelius (dentro de los programas de software propietario) y MuseScore (dentro de los programas software libre).

Entre otros recursos se han integrado en distintos ámbitos, los teclados electrónicos, los pianos digitales, los pianos acústicos con tecnología incorporada (sistemas Silent, dispositivos Disklavier) o los modernos pianos híbridos en los que se han aplicado una serie de innovaciones que les permite

\footnotetext{
${ }^{1}$ Quede constancia del profundo agradecimiento a los diez alumnos de enseñanzas profesionales y a sus familias por su activa disposición en esta experiencia pedagógica, por su implicación, tiempo y entusiasmo.
} 
emular con gran verosimilitud la amplia variedad de matices y texturas de un piano gran cola, tanto en lo que se refiere a calidad sonora, como a la percepción físico/táctil; además de diversos programas destinados a tareas como mejorar el entrenamiento auditivo o la fluidez en la lectura de textos musicales.

$\mathrm{Al}$ igual que en otros entornos educativos, parte del profesorado de los conservatorios ha utilizado las posibilidades que ofrece la red en la elaboración y diseño de webs temáticas, blogs educativos, así como las plataformas educativas, en su mayoría desarrolladas por las administraciones educativas, u otras aplicaciones web de tipo colaborativo como Moodle. Instituciones como la Fundación Albéniz ${ }^{2}$ o la ABRSM ${ }^{3}$ están llevando a cabo diversas iniciativas en la elaboración de materiales, repositorios, recursos on-line y aplicaciones destinadas al desarrollo de objetivos relacionados con el progreso musical. Entre éstas encontramos varias que proponen ejercicios interactivos de reconocimiento de alturas, intervalos, escalas, acordes, ritmos (ABRSM Aural Trainer, Aural G1 for ABRSM), también están aquellas aplicaciones que presentan las partituras convencionales con un refuerzo visual (mediante la proyección de un teclado, como por ejemplo Etude) y otras, que ofrecen la audición de piezas musicales con distintas indicaciones metronómicas (ABRSM Practice Partner). La progresiva digitalización de recursos musicales como partituras, vídeos y registros de sonido (IMSLP, Youtube, Spotify) ha provocado que estos recursos, que tradicionalmente se empleaban en contextos específicos de aprendizaje formal, se utilicen ahora en otros entornos informales de aprendizaje, lo cual tiene efectos de diversos grados y características en las prácticas de aula.

En vista de esto puede ser útil recordar lo planteado por Peter Webster, uno de los principales investigadores en el contexto de la tecnología aplicada al campo de la educación musical, quien destaca el papel creativo en el proceso de aprendizaje del arte con el uso de la tecnología:

La tecnología musical es más que el diseño de un hardware para solucionar un problema de interpretación musical, más que aprender a usar un programa de notación musical. Es más que diseñar una presentación multimedia para una clase de historia de la música o usar un programa de acompañamiento inteligente para ayudar a aprender una obra nueva. Es todas estas cosas, más un modo de relacionarse con la música en un esfuerzo para mejorar la experiencia musical, respetando siempre la integridad de arte (Webster, 2002, citado en Díaz \& Giráldez, 2010, p. 270).

\section{LOS ESTILOS DE APRENDIZAJE EN LA PRÁCTICA DOCENTE MUSICAL}

Las aportaciones de los estilos de aprendizaje abarcan un campo amplio y diverso en el mundo educativo (Salas, 2008). Alonso, Gallego y Honey (1995, p. 48) definen los estilos de aprendizaje

\footnotetext{
2 El Proyecto Magister Musicae de la Fundación Albéniz es una web en la que pueden consultarse un catálogo de enseñanza musical digitalizada de más de tres mil horas con clases impartidas por más de doscientos músicos internacionales. Paloma O`Shea, presidenta de la fundación ha promovido este proyecto, en el que además de la Escuela Superior de Música Reina Sofía también se han implicado otras prestigiosas instituciones de educación musical. Con el apoyo del Ministerio de Ciencia y Tecnología y otros organismos han logrado desarrollar HTCMedia, un potente recurso que facilita la utilización de grandes cantidades de información en vídeo. El contenido de las clases es grabado en las escuelas de música, posteriormente las lecciones son analizadas y seleccionadas pedagógicamente, para luego ser catalogadas por obra y movimientos, en fragmentos de una duración predeterminada. De esta manera, se facilita al usuario el acceso a un contenido o pieza determinado, en primer lugar a través de la selección del instrumento o agrupación y después a través de los criterios profesor o compositor y para finalmente acceder a una pieza o movimiento de la misma.

3 La ABRSM (Associated Board of the Royal Schools of Music) es una institución, originaria del Reino Unido, que ofrece un sistema estandarizado de evaluación y exámenes por niveles en más de noventa países. En la actualidad ofrece varios programas de formación continua a través de cursos, talleres y seminarios, además de contar con un servicio de publicaciones.
} 
como "los rasgos cognitivos, afectivos y fisiológicos que sirven como indicadores relativamente estables, de cómo los discentes perciben, interaccionan y responden a sus ambientes de aprendizaje". Abordar el estudio de los estilos de aprendizaje en contextos musicales implica ineludiblemente estudiar factores como la personalidad, las formas de procesamiento de la información, las estrategias y los períodos de práctica, el entorno y los ambientes, la motivación o la interacción docente-discente, entre otros. En esta sección nos circunscribiremos a las aportaciones de aquellos trabajos que han profundizado en los modelos basados en el procesamiento de la información dentro del contexto de la práctica didáctica pianística, tales como el artículo El piano con corazón (Pérez, 2003); las publicaciones electrónicas Estilos de aprendizaje y educación instrumental (Rosabal, 2008); Strengthening musical memory using the dunn \& dunn learning styles perceptual modalities (Dyvbig \& Church, 2005) y Learning styles and piano teaching (García, 2002). Éstos plantean las categorías en función de los canales sensoriales de recepción de información tipificando el estilo visual, el estilo auditivo y el estilo táctil/kinésico-corporal. Dyvbig y Church distinguen por separado la categoría táctil de la kinésico-corporal. Pérez (2003) establece que en el proceso de aprendizaje musical se produce la interconexión de tres esferas: acústica (en la que se produce el hecho sonoro), motora (donde se asienta la sensación corporal) y visual (donde se desarrollan los procesos racionales del aprendizaje) que interaccionan en la ejecución pianística y en las que además operan los siguientes tipos de memoria: memoria auditiva, memoria motriz, memoria comprensiva o estructural, memoria visual. Entre las aproximaciones más completas a las características de estos perfiles encontramos la descripción de Rosabal (2008) quien nos ofrece ejemplos de músicos célebres con alguno de estos perfiles dominantes o la aportación de García (2002) en su artículo Learning styles and piano teaching:

\begin{abstract}
Los estudiantes de tipo visual piensan en imágenes y suelen transformar cualquier información en dibujos, cuadros o esquemas [...]. Como instrumentistas, estos estudiantes pueden leer a vista bien, pero si se les pide que canten la melodía que acaban de realizar, puede que tengan dificultades. Este estudiante puede llegar a tocar con gran precisión, pero pudiera tener problemas para realizar una interpretación expresiva, así como acometer la memorización de las piezas que estudia. [...] Los estudiantes auditivos aprenden mejor a través del oído, de la escucha. Suelen ser conversadores y puede que tarareen y vocalicen mientras tocan. Puede que se distraigan con facilidad ante cualquier estímulo sonoro y pueden tener dificultades para expresarse por escrito. Estos alumnos pueden tocar cualquier tipo de pieza exclusivamente de oído y se suelen frustrar a la hora de leer la música escrita. Prefieren adivinar una melodía, pieza, sección y escucharla varias veces en lugar de leerla [...]. El alumno, con un perfil táctil/kinésico-corporal, es aquel que tiende a aprender a través de las sensaciones que registra en su cuerpo. Estos alumnos aprenden mejor "haciendo". Sentir el movimiento del cuerpo, del gesto, desde una perspectiva global se convierte en su método preferido. Son alumnos que se benefician de los materiales que planteen un formato de juego, de forma que ellos mismos los puedan manipular [...] (García, 2002, pp. 1-2).
\end{abstract}

La correcta evaluación de estas características se revela como una de las herramientas más eficaces para que los profesores puedan establecer la selección y planificación de tareas más adecuadas para cada alumno. De la misma forma, el propio autoconocimiento del profesor, de su propio estilo de aprendizaje le permitirá por una parte, conocer mucho mejor los aspectos positivos y negativos de su forma de enseñar, y como consecuencia de ello, le brindará nuevas opciones metodológicas y recursos más efectivos para realizar valoraciones más ajustadas en las que se pueda controlar en cierta medida los sesgos que pueden distorsionar la percepción de su actividad, sea ésta musical o pedagógica. Con respecto a esto, no debemos olvidar, que la relación profesor-alumno necesita de una observación y cuidado constantes, como relación humana que es, no exenta de problemas y con posibilidad de desarrollar distintos grados y/o tipos de disfuncionalidad. El conocimiento de uno mismo y de su propio estilo es un indicador de madurez personal, que puede evitar muchos conflictos y ayudar, desde una perspectiva indudablemente emocional, a reconducir las situaciones de dificultad o estancamiento, que inevitablemente surgen a lo largo del proceso de aprendizaje. Seguido de estas líneas se expone la sugerencia de Violeta Hemsy de Gainza (2003) en una de sus experiencias formativas con jóvenes profesores: 
Comenzó solicitando a los participantes que se procuraran papel y lápiz con el objeto de escribir una "carta de amor” a su respectivo instrumento, sin nombrarlo, aprovechando para decirle todo lo que sentían por él y desearían transmitirle. Cuando terminaron la tarea propuesta algunos leyeron en voz alta sus misivas y luego se integraron en grupos de ocho o diez personas con el fin de procesar en forma colectiva los aportes individuales (Hemsy de Gainza, 2003, p. 18).

Esta autora advierte la necesidad de una continua reflexión sobre la propia práctica docente y no solo desde la perspectiva del conocimiento técnico, musical o de estilo de pensamiento/aprendizaje, sino desde la perspectiva de la propia experiencia individual y en cómo ésta influye en el desarrollo personal y profesional del docente, esto es, cómo evoluciona el vínculo que el mismo establece con la música a lo largo de su proyecto vital.

\section{PLANTEAMIENTO DEL PROBLEMA}

La propuesta que se presenta a continuación, es una actividad de vídeo-grabación que forma parte de un proyecto de investigación-acción consistente en el estudio cualitativo de casos con el objetivo de observar, describir y detectar los estilos de aprendizaje más característicos de un grupo de estudiantes de conservatorio de la especialidad de piano. En esta práctica, hemos propuesto a los alumnos registrar en vídeo una situación de aprendizaje: un período de estudio breve, de diez a quince minutos, para observar las conductas, actitudes y estrategias, en relación tanto a los estilos de aprendizaje como a la valoración de la calidad de la práctica instrumental orientada al progreso de sus habilidades musicales.

El progreso en la práctica musical instrumental, para que sea eficaz, es preciso que esté sustentado por estrategias de prácticas instrumental efectivas. Algunos autores como Tripiana (2012) las definen de la siguiente manera:

Aquellos pensamientos y comportamientos que, de forma consciente e intencional, guían al intérprete durante la práctica deliberada de su instrumento e intervienen en el modo en el que selecciona, organiza, procesa, integra y ejecuta sus conocimientos y habilidades musicales; en su estado emocional; y/o en su motivación; con el propósito de adquirir, almacenar y posteriormente poder reproducir resultados instrumentales, a su juicio, positivos y en el menor tiempo de consecución posible (Tripiana, 2012, p. 71)

La observación de conductas y estrategias es uno de los temas que más interés suscita en la actualidad, tal y como puede comprobarse en el contenido del IV Reflective Conservatorie Conference: Creativity and changing cultures 2015, donde se muestra un número significativo de investigaciones centradas en el estudio de los factores implícitos del aprendizaje y que utilizan las vídeo-grabaciones como instrumentos reguladores de la práctica musical. Como ejemplo de esto, encontramos el trabajo presentado por Carlos López-Real (2015): Vídeo as a tool for self-regulation combining the power of technology with Liz Lerman`s critical response process.

Desarrollar este tipo de prácticas requiere:

- Planificación de tareas para conseguir fines concretos.

- Concentración en la realización correcta de esas tareas.

- Motivación y esfuerzo (psíquico y físico) para abordar esas tareas y mantener la atención a lo largo del proceso.

- Realización regular y sistemática, a lo largo de un período de tiempo suficiente, como para que pueda confirmarse un grado de desarrollo de la habilidad que se pretende observar.

\section{MÉTODO}

La práctica que se propone utiliza como herramienta de diagnóstico la vídeo-grabación. El objetivo de la misma ha sido observar pautas de conducta y estrategias durante un período determinado de estudio y ante una propuesta de repertorio, a priori nueva para los estudiantes. 


\section{Muestra}

La actividad que aquí se presenta tuvo lugar en la finalización del curso 2010/11, momento en el que los diez casos objeto de estudio presentaban las siguientes características en lo que respecta a curso de enseñanzas profesionales y edad, tal como figura en la tabla que aparece a continuación. Para acometer la descripción de los datos recogidos en cada uno de los casos y preservar la identidad de los participantes se asignó un identificador a cada alumno y alumna. Para los alumnos se utilizaron nombres de reconocidos intérpretes y compositores y para las alumnas nombres de compositoras.

\begin{tabular}{|l|c|c|}
\hline \multicolumn{1}{|c|}{ Identificador } & $\begin{array}{c}\text { Curso de enseñanzas } \\
\text { profesionales }\end{array}$ & Edad \\
\hline Mariana Martines & $1^{\circ} \mathrm{EP}$ & 11 \\
\hline Art Tatum & $1^{\circ} \mathrm{EP}$ & 13 \\
\hline Fanny Hensel & $1^{\circ} \mathrm{EP}$ & 13 \\
\hline Karl Tausig & $1^{\circ} \mathrm{EP}$ & 12 \\
\hline Friedrich Kalkbrenner & $1^{\circ} \mathrm{EP}$ & 13 \\
\hline Johann Nepomuk Hummel & $1^{\circ} \mathrm{EP}$ & 14 \\
\hline Maddalena Casulana & $2^{\circ} \mathrm{EP}$ & 12 \\
\hline Clara Wieck & $2^{\circ} \mathrm{EP}$ & 13 \\
\hline Keith Jarret & $2^{\circ} \mathrm{EP}$ & 15 \\
\hline Lousie Adolpha LeBeau & $2^{\circ} \mathrm{EP}$ & 15 \\
\hline
\end{tabular}

Tabla 1: Cuadro de datos de alumnos: identificador, curso de enseñanzas profesionales y edad

\section{Instrumentos: La grabación didáctica de una práctica de estudio}

El objetivo fundamental de esta actividad fue detectar “cómo estudia el alumno”, qué mecanismos utiliza y qué tipo de respuestas manifiesta. Con esta vídeo-grabación se pretendió registrar los primeros momentos de acercamiento a una pieza/s nueva/s, ya que la propuesta planteada constaba de obras no conocidas por el grupo de alumnos, desde un punto de vista técnico. Con ello se procedió después a observar las acciones, conductas y actitudes que se manifiestaron a lo largo del período de grabación, en definitiva, una situación de estudio en un contexto real y definido. Además de las descripciones señaladas, el material recopilado admitía ser contrastado con otra información relevante, como otros registros de audio o vídeo, otras producciones de esos alumnos e incluso distintos documentos de evaluación. En el siguiente cuadro explicativo se muestran los objetivos de observación aplicados:

\begin{tabular}{|l|l|}
\hline $\begin{array}{l}\text { Objetivos de } \\
\text { observación }\end{array}$ & $\begin{array}{l}\text { Identificar las conductas, actitudes y acciones que realizan los alumnos cuando abordan el inicio de } \\
\text { una pieza. } \\
\text { Registrar las reacciones e impresiones que registran en ese proceso. } \\
\text { Reconocer la capacidad de resolución en las tareas propuestas. }\end{array}$ \\
\hline ¿Qué se observa? & $\begin{array}{l}\text { Grado de resolución e independencia en la ejecución de tareas. } \\
\text { Tipos de recursos que utilizan los alumnos: auditivo, de lectura, de memorización, de reflexión, } \\
\text { seguimiento de pautas, u otros. } \\
\text { Reacciones emocionales frente a las posibles dificultades y a la propia experiencia. } \\
\text { Capacidad de mantenimiento de la atención en las tareas que realicen. } \\
\text { Evidencia de comportamientos o actitudes relacionados con los estilos de aprendizaje pianísticos: } \\
\text { visual, auditivo o táctil/kinésico-corporal. }\end{array}$ \\
\hline ¿Para qué se \\
observa? & $\begin{array}{l}\text { Establecer similitudes y/o diferencias entre lo que los alumnos "hacen” y lo que “dicen que hacen” } \\
\text { Contrastar datos e información con otros instrumentos: grabaciones de otras situaciones de } \\
\text { aprendizaje, datos académicos, cuestionarios u otras producciones de los alumnos. } \\
\text { Detectar canales y modalidades preferentes de aprendizaje. }\end{array}$ \\
\hline
\end{tabular}

Tabla 2: Objetivos de observación de la práctica de estudio 


\section{Recursos}

Para acometer esta práctica fue necesario asegurarse de que los alumnos dispusiesen de los recursos necesarios para llevarla a cabo. Por ello se procedió previamente a explicar la estructura de la actividad (tiempos, recursos, espacios, etc.), el contenido de la misma (con el grado de profundidad que cada caso requiriese), y se facilitaron una serie de elementos (lápiz, goma, metrónomo, dispositivo de grabación) así como una breve guía didáctica de estrategias de práctica, de la que podrían disponer una vez finalizada la grabación.

\section{Procedimiento}

- Se presentó la siguiente selección de piezas de diferentes estilos y características, con sus correspondientes materiales complementarios:

\begin{tabular}{|c|c|}
\hline Planteamiento didáctico de repertorio & Materiales \\
\hline P. D. 1. sobre el tema moon river de Henry Mancini & Partituras de dos versiones del tema y guía didáctica. \\
\hline $\begin{array}{c}\text { P. D. 2. del estudio L'avalanche de Stephen Heller } \\
\text { P. D 3. Del Andante Grazioso de Wolfgang A. } \\
\text { Mozart }\end{array}$ & Partitura completa y guía didáctica. \\
\hline $\begin{array}{c}\text { P. D. 4. De la pieza Laid Back Blues de Pamela } \\
\text { Wedgwood }\end{array}$ & Partitura completa del movimiento y guía didáctica. \\
\hline
\end{tabular}

Tabla 3: Propuestas didácticas para la práctica de estudio

A continuación se siguió la siguiente secuencia de tareas:

- El profesor ofrece un dossier con todo el repertorio propuesto y sus materiales complementarios.

- El alumno elige una o dos piezas para tratar de resolverla/s en un tiempo determinado (10 -15 minutos).

- El profesor emite las explicaciones oportunas a través de una guía didáctica de estrategias (material complementario) de cada una de las piezas propuestas y explica todo aquello demandado por el alumno. Para ello acomete las siguientes actuaciones: pregunta por las preferencias del repertorio, explica características de las piezas, ayuda en la identificación de dificultades, ejecuta fragmentos musicales, destaca estrategias de práctica, realiza preguntas didácticas, solicita información sobre los planes que el alumno tiene pensado llevar a cabo en su tiempo de estudio en esta práctica (10 minutos).

- La grabación se desarrolla en un contexto privado de estudio (en este caso ha sido un aula), los alumnos realizan la misma sin la presencia ni intervención del profesor. La observación que se propone es por tanto, directa no participante.

- El alumno puede hacer uso de las partituras, guías didácticas y materiales tal y como considere necesario.

- Al finalizar la actividad de grabación, el alumno dispone de la totalidad de los materiales diseñados para esta actividad (las piezas completas y sus materiales complementarios en este caso, guías didácticas y versiones) para su utilización particular posterior, si así lo desea.

- Una vez finalizada la grabación, se procede realizar una descripción de lo acontecido en función de los aspectos especificados en el cuadro “Objetivos de observación” de manera que se adjuntan al estudio individual de cada caso. 


\section{Características de las guías didácticas de tareas}

La planificación de estas grabaciones ha incluido el diseño de cuatro guías didácticas de tareas que se han utilizado tanto en las explicaciones previas a la actividad de grabación como durante la grabación en sí. En estos materiales se ha procurado elaborar un planteamiento didáctico específico y claro, con indicaciones de fácil comprensión y sugerencias viables al momento de realizar la práctica de estudio y también posteriormente a ella. Uno de los retos que de hecho ha planteado esto, ha sido crear distintos tipos de exposiciones, preguntas e instrucciones para una práctica autónoma e independiente, que se adapte a su vez también al estilo de cada alumno, mediante la propuesta de distintos formatos de preguntas, instrucciones y actuaciones. De la misma forma, en estos materiales se han añadido varias referencias web con el objetivo de facilitar recursos audiovisuales, que puedan ser consultados en cualquier momento posterior a la realización de esta actividad. Intencionalmente, en las propuestas 1 y 3 no figuran los nombres de los autores/compositores, ya que se ha utilizado esta circunstancia como un elemento a partir del cual plantear una pregunta didáctica en la sección previa explicativa a la sesión de grabación. Por último, queda indicar que las actividades propuestas en los materiales didácticos incluyen una cantidad de tareas superior a lo que puede realizarse en la sesión de grabación. Con esto se ha pretendido promover un doble objetivo. Por una parte, detectar qué estrategias preferentes manifiestan los alumnos ante nuestra propuesta y por otra, ofrecer a los alumnos herramientas o medios que les faciliten o ayuden en una práctica a posteriori, esto es, que esta actividad tenga una repercusión más allá de lo acontecido en su momento de realización. En los anexos de este artículo se exponen los extractos más representativos de estas guías didácticas.

\section{RESULTADOS}

Se emiten las consideraciones más significativas observadas así como los datos recopilados tras la realización de las diez vídeo-grabaciones:

- Tiempo y ambiente: Casi todas las grabaciones se ajustaron al tiempo establecido. Solamente un caso registró menos tiempo del requerido (9'50’) y dos casos superaron ligeramente los quince minutos (15'43” y 16'28'”). Sí puede resultar significativo que el caso que registró el menor tiempo de estudio ha mostrado conductas de baja motivación en los estudios musicales. En cuanto al ambiente, las tareas realizadas por los alumnos se desarrollaron con normalidad, obviamente con niveles de concentración, atención y resultados variables. No obstante no debemos olvidar que cualquier proceso de grabación produce en quien es grabado varias emociones derivadas de sentirse observado: cierta incomodidad, sensación de vivir una realidad poco natural, preocupación por lo que se muestra; aspectos, éstos, perceptibles sobre todo al inicio del proceso, observándose pequeños matices o diferencias en la respuesta de adaptación a esta "nueva situación de aprendizaje”. Aunque al inicio algunos pudiesen mostrar cierta incomodidad, sí se detectó que varios de ellos intensificaron la atención en determinadas tareas. Todos los participantes realizaron ejecuciones parciales o completas de las dos piezas elegidas, aunque casi todos centraron su actividad considerablemente más en una de ellas.

- Contenido de la grabación: La elección de las piezas para realizar la grabación reveló aspectos relevantes relacionados con el gusto musical. Durante las explicaciones previas a la grabación, se ha constatado que la mayoría de los alumnos participantes desconocían el concepto "estilo musical”, entendido éste como el conjunto de modelos reproducidos en un grupo de obras, con una serie de características relacionadas entre sí. En algunos momentos se constató que varios alumnos son capaces de establecer diferencias entre algunos tipos de obras y piezas de las que tocan, pero manifiestan muchas dificultades si las piezas se descontextualizan de su período histórico. También se observó no utilizan con seguridad los términos estilo musical, género musical ni forma musical. Se expone un gráfico con las elecciones realizadas: 


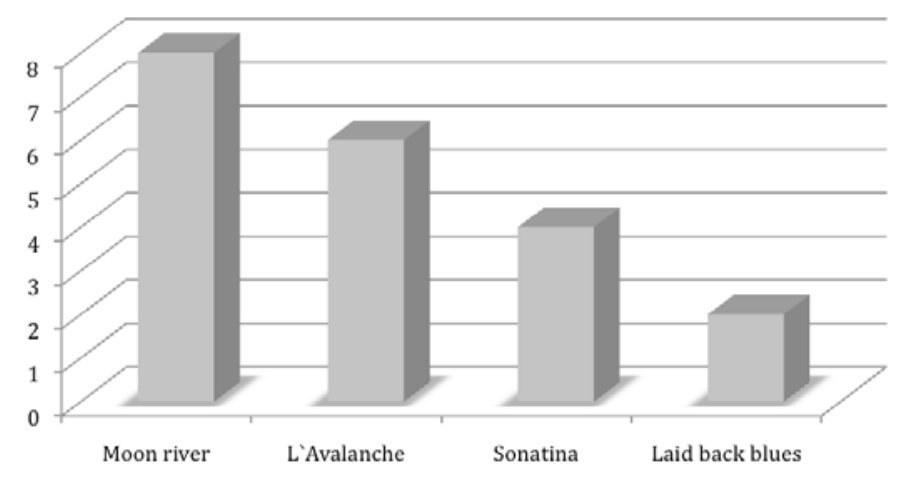

Fig. 8. Gráfica de las piezas seleccionadas por os alumnos para la práctica de estudio vídeo-grabada.

- Relación estrategias y rendimiento: En relación a este aspecto se detectó que, tanto la variedad como la efectividad de las estrategias de práctica utilizadas por los alumnos se encuentran profundamente relacionadas con su nivel de destreza instrumental. En aquellos casos cuyo rendimiento es óptimo, esto es, calificaciones altas, práctica de estudio regular; las tareas observadas parecen no haber supuesto un especial esfuerzo y se han caracterizado por un desarrollo fluido. De manera gradual, a medida que las habilidades (sean de contenido o procedimiento) están menos desarrolladas, los alumnos han tenido que poner en marcha estrategias facilitadoras, haciéndose evidente una falta de fluidez en la realización de las tareas emprendidas.

- Materiales y recursos utilizados: En este punto exponemos uno de los datos más significativos de la experiencia. En todas las grabaciones realizadas, tanto el uso de los materiales (lápiz, metrónomo) como el seguimiento de las indicaciones de las guías de tareas se han restringido al mínimo (no se completó ninguna propuesta, aunque varios lograran ejecutar aceptablemente partes de las piezas elegidas). Esto nos hace pensar que el uso de estrategias de práctica en estos niveles está profundamente relacionado con las prácticas de estudio individual. Otro aspecto que se registró es que varios alumnos, en las explicaciones previas a la grabación, manifestaron interés en poner en práctica determinados tipos de tareas, que luego en la grabación no realizaron. Esto pone en evidencia la importancia que tienen los contextos de práctica informal en el desarrollo de las habilidades musicales. La observación de las prácticas formales e informales son una de las prioridades que en estas enseñanzas deberían acometerse. Se ha detectado que en la mayor parte de los casos, la frontera entre practicar y estudiar no está claramente definida, lo cual puede dificultar o ralentizar el progreso de determinados aspectos o parámetros asociados a las habilidades instrumentales (realización adecuada del gesto, digitaciones coherentes, atención y consecución de planos sonoros, por citar algunos aspectos). La mayor parte del tiempo grabado está formado por ejecuciones de fragmentos musicales. Se han registrado relativamente pocos períodos de tiempo donde se pudiese constatar que los alumnos están pensando o reflexionando sobre lo que van a hacer. En los momentos en los que esto se ha dado, ha coincidido con aquellos alumnos de mayor edad, los cuales han mostrado en la grabación períodos de reflexión, tales como la lectura de los textos musicales o las indicaciones de práctica. En algunas de las grabaciones puede verse que aunque sentados frente al instrumento, no tocan, leen sobre el atril o realizan acciones tales como gestos sobre el teclado sin que el instrumento suene.

- Estilos de aprendizaje: Las estrategias utilizadas por los alumnos en estas grabaciones han revelado pautas de actuación profundamente relacionadas con los estilos de aprendizaje visual, auditivo y táctil/kinésico-corporal. García (2002) explica que el desarrollo equilibrado de los tres estilos es un indicador de desarrollo de las habilidades musicales, aspecto éste que se ha puesto también de manifiesto en las grabaciones registradas. En la gráfica que se muestra a continuación puede apreciarse que los alumnos de mayor edad manifiestan características de los tres estilos. Esto también se evidencia en el caso de la alumna de menor edad (martines, 11 años), la cual es además un caso que representa una circunstancia de cierta excepcionalidad, ya que es una alumna que se 
encuentra matriculada en un curso superior al que le correspondería por su edad y que además presenta un rendimiento alto, con altas calificaciones.

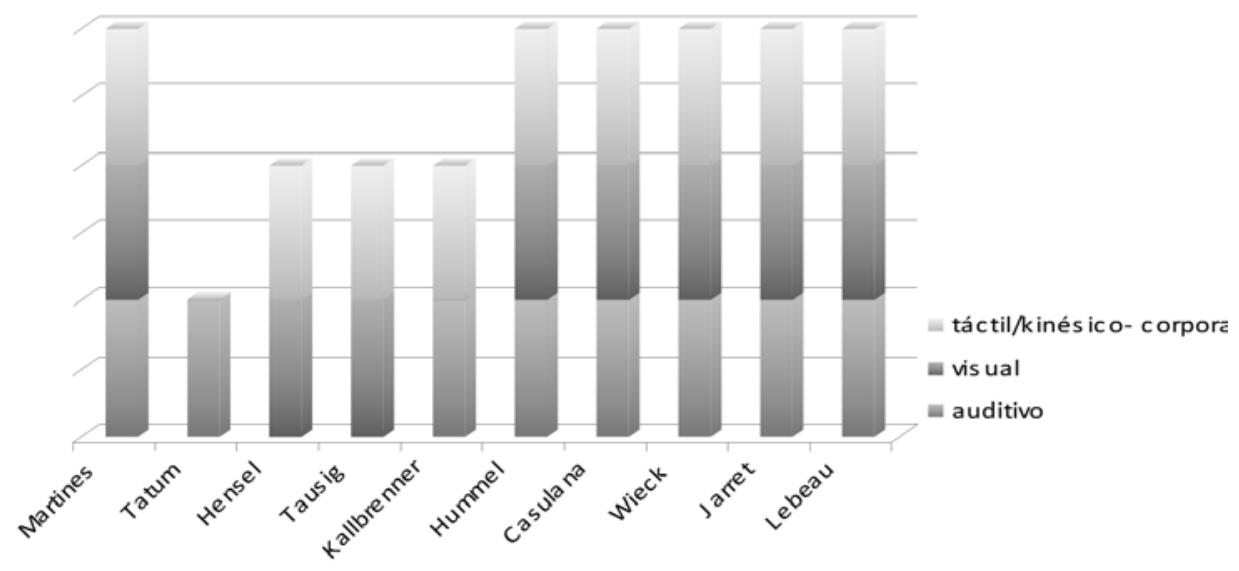

Fig. 9. Estilos de aprendizaje observados en la práctica de estudio vídeo-grabada.

- Acciones relacionadas con el aprendizaje y las respuestas emocionales: Las principales conductas observadas han sido la realización de tareas ejecutivas: tocar, poner en práctica estrategias concretas (manos separadas, por secciones cortas); dirigir corporalmente el pulso, verificar la posición en el teclado (movimientos viso-motores); experimentar con la sonoridad (utilizar el pedal); y en menor grado pensar, reflexionar, solfear, leer y escribir anotaciones. En cuanto a las respuestas emocionales, se han observado y percibido comportamientos diversos: ligero nerviosismo al principio, cierta ansiedad manifestada a través del movimiento corporal no vinculado necesariamente a la acción de aprendizaje o ante determinadas dificultades (realización de una digitación), cierta frialdad y concentración en determinadas acciones, disfrute y experimentación con las sonoridades obtenidas, e incluso en algunos casos han sido perceptibles momentos de aburrimiento, cuando el alumno o alumna estimaba que los objetivos de la tarea se habían conseguido.

La realización de esta grabación didáctica ha hecho visible una variedad de acciones ante una propuesta de tareas común. Aunque en principio se pudiera considerar que determinadas conductas o actuaciones son coincidentes, existen matices diferenciadores que hacen que percibamos que en cada una de ellas se revela la singularidad de cada alumno. Un ejemplo de esto es cómo podemos interpretar una determinada acción efectuada por un alumno o alumna. Una forma de proceder, como mover el cuerpo mientras se ejecuta un fragmento musical, puede ser interpretada como un síntoma ligero de ansiedad o también como una manera en el que el aprendiz trata de controlar el ritmo o la expresividad de su ejecución.

Por otra parte, esta experiencia abre nuevas posibilidades al diseño de otras actividades con implicaciones directas tanto en el aprendizaje de los alumnos como sobre la propia práctica docente, ya que permite:

- Detectar las fortalezas y debilidades de la práctica instrumental.

- Proponer prácticas musicales vinculadas con las necesidades y las dificultades de aprendizaje.

- Diversificar las tareas diseñadas para un objetivo concreto.

- Establecer indicadores de progreso en habilidades concretas: lectura a vista, improvisación, técnica e interpretación.

- Habilitar herramientas que favorezcan los procesos de reflexión y de auto-evaluación del profesor y del alumnado.

- Desarrollar estrategias metacognitivas de los aspectos menos tangibles de la práctica instrumental, así como el auto-conocimiento personal de los alumnos. 
- Crear recursos adicionales para los docentes, que permitan obtener información fiable sobre los aspectos “ocultos” del aprendizaje: detección de incidencias, recopilación y contraste de información, mejora del diseño de registros.

\section{CONCLUSIONES}

La realización de esta actividad ha supuesto una oportunidad para valorar varios aspectos no sólo de las prácticas de enseñanza aprendizaje, sino también de otros factores implícitos en la práctica musical que pueden pasar inadvertidos para una valoración adecuada del progreso musical, como son el grado de dominio de las estrategias de práctica, la implicación real con las piezas de estudio que se han propuesto, el grado de atención y concentración que pueden mantener los alumnos. Al margen de las consideraciones particulares que esta práctica de grabación tiene para el estudio de cada caso con sus circunstancias específicas y la magnífica oportunidad que ofrece para valorar aspectos relacionados con el formato y contenido didáctico de la misma, el objetivo de este artículo ha sido explicar los resultados más significativos referidos a la aplicación de este recurso TIC, la grabación didáctica como práctica docente. Obvio es que parte de la validez y eficacia de esta práctica se relaciona con la combinación y el uso de otros instrumentos de investigación y que, como estudio cualitativo de diez estudios de caso, los resultados derivados se limitan a sí mismos, no pudiendo analizarse la problemática que plantean en un marco más amplio, lo cual podría y debería ser estudiado.

La observación sistemática de los alumnos en su momento de estudio proporciona información muy importante, ya no sólo de cómo ellos perciben y aprenden, sino de cómo nosotros enseñamos, qué tipos de referencias ofrecemos y hasta qué punto incluso, resultamos convincentes. En este sentido, reflexionar sobre lo que enseñamos de una manera explícita y relacionarlo con lo que los alumnos manifiestan que aprenden de una manera implícita debería ser una línea prioritaria de la práctica docente y de investigación en los contextos de enseñanza musical especializada. Ello nos llevará ineludiblemente a comprender mejor nuestra manera de enseñar, en definitiva nuestro estilo de enseñanza, convirtiendo la mejora profesional en algo que sin duda contribuirá positivamente en nuestro plano más humano y personal.

\section{Referencias}

Associated Board of the Royal Schools of Music (sf). Practice tools and apps. Consultado en http://gb.abrsm.org/en/exam-support/practice-tools-and-applications/ el 15/09/2014.

Alonso C. M., Gallego D. J., \& Honey P. (1995). Los estilos de aprendizaje: Procedimientos de diagnóstico y mejora. Bilbao: Mensajero.

Brendel, A. (2013): De la A a la Z de un pianista. Un libro para amantes del piano. Barcelona: Acantilado.

Díaz, M. \& Giráldez, A. (Eds.) (2010): Aportaciones teóricas y metodológicas a la educación musical. Una selección de autores relevantes. Barcelona: Graó.

Dybvig, T. (2005). Strengthening musical memory using the dunn \& dunn learning styles perceptual modalities. National Conference on Keyboard Pedagogy in Oakbrook, IL. Consultado en http://www.teresadybvig.com/Dybvig-poster.pdf el 18/09/2011.

García, S (2002). Learning styles in piano teaching. Piano Pedagogy Forum, 5(1). Consultado en http://www.mtnacertification.org/media/58356/Garcia Article.pdf el 11/02/2011.

Heller, S (1942): L’Avalanche, Estudio op. 45 n² 2. Nueva York: Schirmer. (Primera publicación, 1845). 
Heller, S (1997): L'Avalanche, estudio $n^{\circ} 2$ op. 45. Partitura pedagógica [Keith Snell]. San Diego, California: Neil A. Kjos. Music Company. (Primera publicación, 1845).

Hemsy de Gainza, V. (2002): Música: Amor y conflicto: Diez estudios de psicopedagogía musical. Buenos Aires: Lumen Humanitas.

Mancini, H (2010): Moon river (Primera publicación 1961) [Arreglo de White Admiral]. Consultado en http://www.pianoplateau.com/content/moon-river-henry-mancini el 11/02/2011.

Mancini, H (1984): Moon river (Primera publicación 1961) [Arreglo propiedad de Famous Music Corporation]. Consultado en: www.sheetmusicplus.com el 11/02/2011.

Mozart, W. A. (1958): Sonatina Vienesa nº 4. Madrid: Real Musical. (Primera publicación, 1783).

Pérez, M (2003). El piano con corazón. Quodlibet. Revista de Especialización Musical, 27, 71-97.

López-Real, C. (2015). Video as a tool for self-regulation combining the power of technology with Liz Lerman`s critical response process. IV Reflective Conservatorie Conference 2015: Creativity and changing cultures (p. 163). Consultado en http://www.gsmd.ac.uk/fileadmin/user_upload/files/Research/Reflective_Conservatoire_201 5/Reflective_Conservatoire_2015_-_full_reader_FINAL.pdf el 21/03/2015.

Rosabal, G (2008). Estilos de aprendizaje y educación instrumental. La Retreta, I(2). Consultado en http://laretreta.net/0102/articulos/estilosdeaprendizaje.html el 2/03/2011.

Salas, R. E. (2008): Estilos de aprendizaje a la luz de la neurociencia. Bogotá: Cooperativa Editorial Magisterio.

Tripiana, S (2012). Estrategias eficaces de práctica instrumental. Música y Educación, 25(91), 64-71.

Wedgwood, P. (1998): Laid-back blues. Jazzin'about fun pieces for piano/keyboard. Londres: Faber Music. 


\section{ANEXOS: EXTRACTOS DE LAS GUÍAS DIDÁCTICAS}

\section{Anexo 1. Extracto de la propuesta del tema "Moon river"}

Partimos de un arreglo sencillo para piano del famoso tema de la película Desayuno con diamantes. Se trata de una melodía en la tonalidad de do mayor, que presenta un acompañamiento basado en una secuencia armónica sencilla. Dispones de dos versiones, una más elaborada y otra más sencilla pero con los elementos básicos de ritmo, melodía y armonía comunes. La versión más elaborada es la que figura a continuación: http://www.youtube.com/watch?v=mEIS9ezCppw.

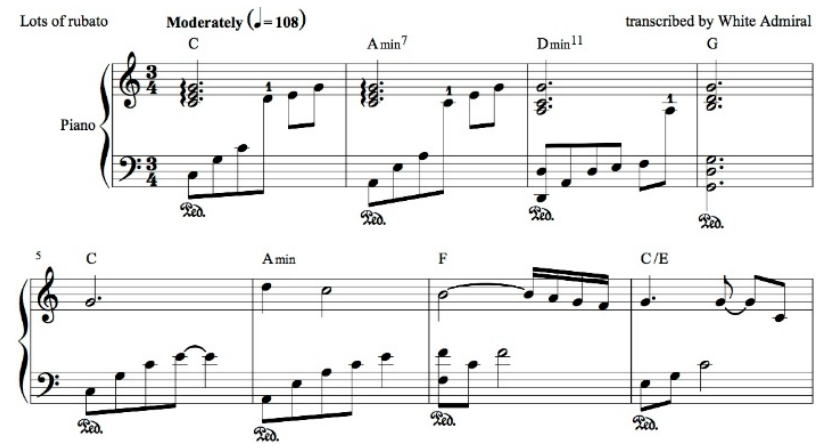

Fig. 1: Tema Moon River en la versión de W. Admiral

En ambos textos encontramos indicaciones de tipo expresivo (ligaduras de fraseo, indicaciones dinámicas y alguna indicación agógica). Los cambios en el discurso armónico están señalados mediante el cifrado americano. A continuación puedes ver la versión más sencilla, pero con los mismos elementos.

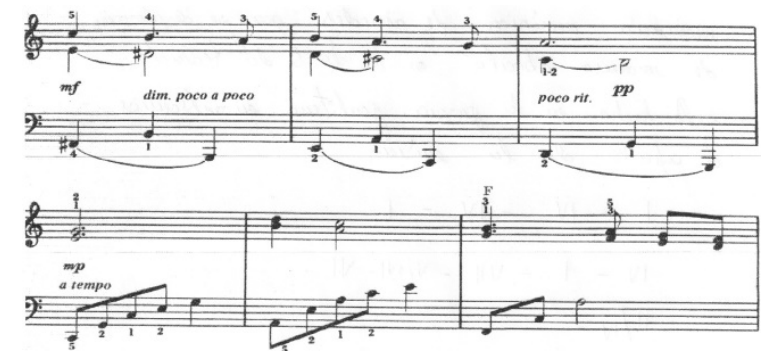

Fig. 2: Fragmento del tema en la versión facilitada

Este tema juega melódicamente con notas de pasos y retardos. Si comparas las dos versiones observarás fácilmente este tipo de notas de adorno.

- ¿Qué notas de paso y adornos encuentras en ambas versiones?

Escucha y lee la siguiente versión jazz: http://www.youtube.com/watch?v=IO0FextfSsM. 


\section{Anexo 2. Extracto de la propuesta didáctica del estudio "L'Avalanche”}

Esta pieza es la número dos de la colección de estudios op. 45. Los estudios de Heller, como los de Bertini, también tienen como objetivo desarrollar la capacidad expresiva. La partitura expone muchas indicaciones de tipo dinámico, expresivo, agógico y de articulación. El tempo Allegro vivace es otro de los retos de ejecución que deben abordarse.

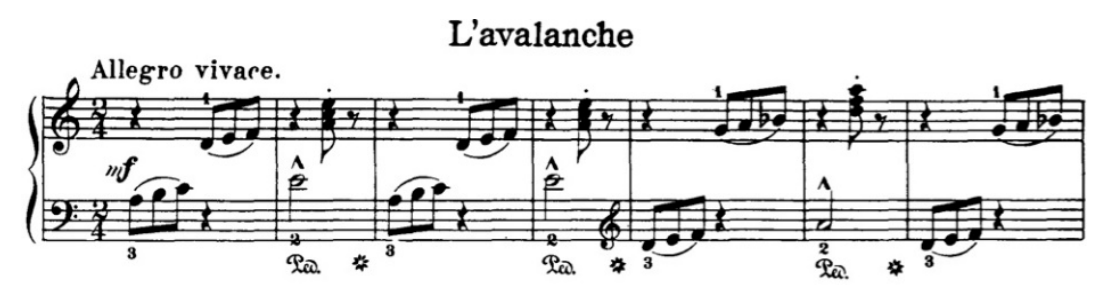

Fig. 3: Fragmento del estudio $n^{\circ} 2$ op. 45 de Heller

Realiza la ejecución de esta pieza, con la partitura anexa (versión Keith Snell, 1997) y si lo consideras necesario aborda estas preguntas y consejos:

- ¿En qué tonalidad está esta pieza? ¿Cuántos tipos de escritura instrumental encuentras?

- ¿En cuántas partes dividirías esta pieza?

- ¿¿Comprendes el significado de todas las indicaciones? ¿Qué significa “poco meno mosso”?

Otras indicaciones que te pueden resultar de utilidad son:

- Revisa los cambios de clave y de posición.

- Observa las ligaduras de expresión.

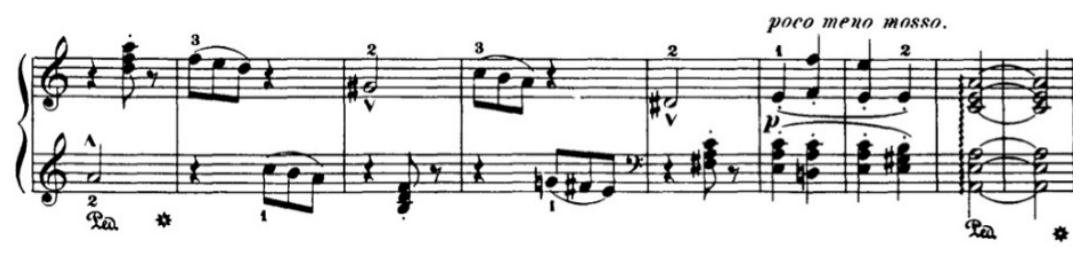

Fig. 4: Fragmento del estudio de Heller con indicaciones expresivas

Puedes escuchar este estudio en este enlace: http://www.youtube.com/watch?v=MRErWum Mbbc. 


\section{Anexo 3. Extracto de la propuesta didáctica del "Andante grazioso" de la cuarta sonatina vienesa}

La pieza clásica elegida reúne de una manera muy clara muchos elementos de la escritura de un compositor muy famoso. La escritura aparentemente fácil que presenta puede confundir al ejecutante. La expresión, en este caso, se encuentra bastante condicionada por los criterios de estilo y ha de conseguirse por medios sutiles, que implican un análisis minucioso del texto. La búsqueda de un tempo adecuado al ritmo del intérprete por una parte y la realización correcta de la variedad de figuraciones en este compás de compasillo son dificultades que, aunque no lo parezca al principio, es necesario trabajar.

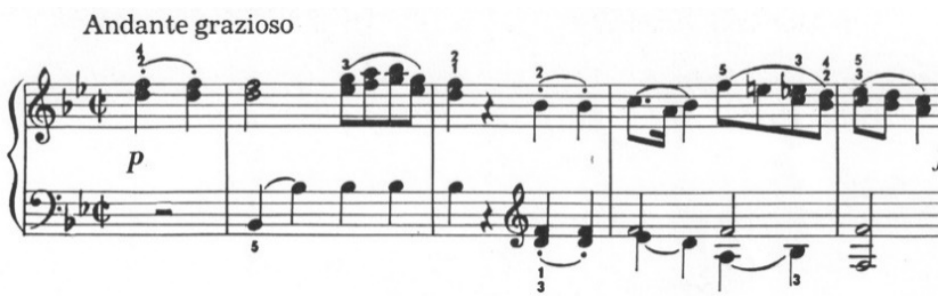

Fig. 5: Cabeza del tema de la cuarta sonatina

Realiza la ejecución de esta pieza y si lo consideras necesario aborda estas preguntas y consejos:

- Selecciona las frases y semifrases. ¿Existen similitudes y diferencias?

- ¿Cómo sientes internamente esas frases?

- ¿En qué secciones dividirías la pieza? ¿Qué tipos de figuras observas?

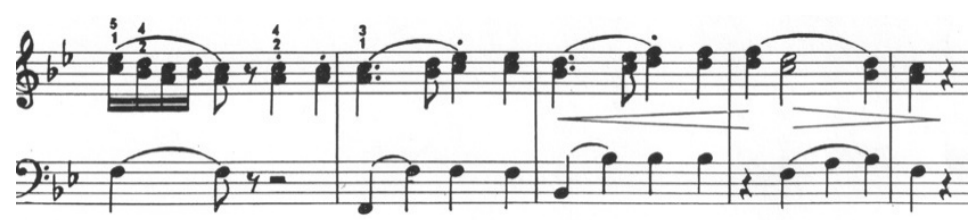

Fig. 6: Fragmento del primer movimiento

Otras recomendaciones previas a la ejecución, que te pueden ayudar:

- Reflexiona sobre qué unidad rítmica vas a tener en cuenta para mantener la regularidad del pulso a lo largo de la ejecución.

- Interioriza las melodías, canta internamente antes de tocar.

- Si no estás familiarizado con la tonalidad, toca la escala de si bemol mayor un par de veces.

- Localiza los fragmentos que consideres más difíciles rítmica y armónicamente.

- Trata de tocar en sincronía con tu propia respiración.

- Realiza una primera lectura sin ornamentos, pero manteniendo el rigor de la medida. 


\section{Anexo 4. Extracto de la propuesta didáctica de la pieza “Laid-back blues” de la colección Jazzin' About}

Las piezas de esta autora cuentan con varios elementos de estilos contemporáneos como el rock, el blues o el jazz. Algunas características de esta pieza son:

- Uso de temas y melodías breves, que pueden desarrollarse en mayor o menor medida.

- Utilización de una breve secuencia de acordes, que se repiten a lo largo de la pieza. En algunos casos, utiliza armonías sofisticadas que incluyen el uso de distintos acordes de séptimas y otros acordes utilizados en el cifrado americano.

- Utilización de barras de repetición, y gran cantidad de indicaciones de tipo expresivo y de articulación.

- Aplicación graduada de patrones rítmicos complejos, en los que se incluyen: síncopas, ritmos a contratiempo, ligaduras, cambios de compás y de tempo.

- Indicaciones de otros tipos de recursos: carácter, equivalencias de pulso, pedalizaciones, indicaciones de gesto/ataque, expresión o cambio de tempo.
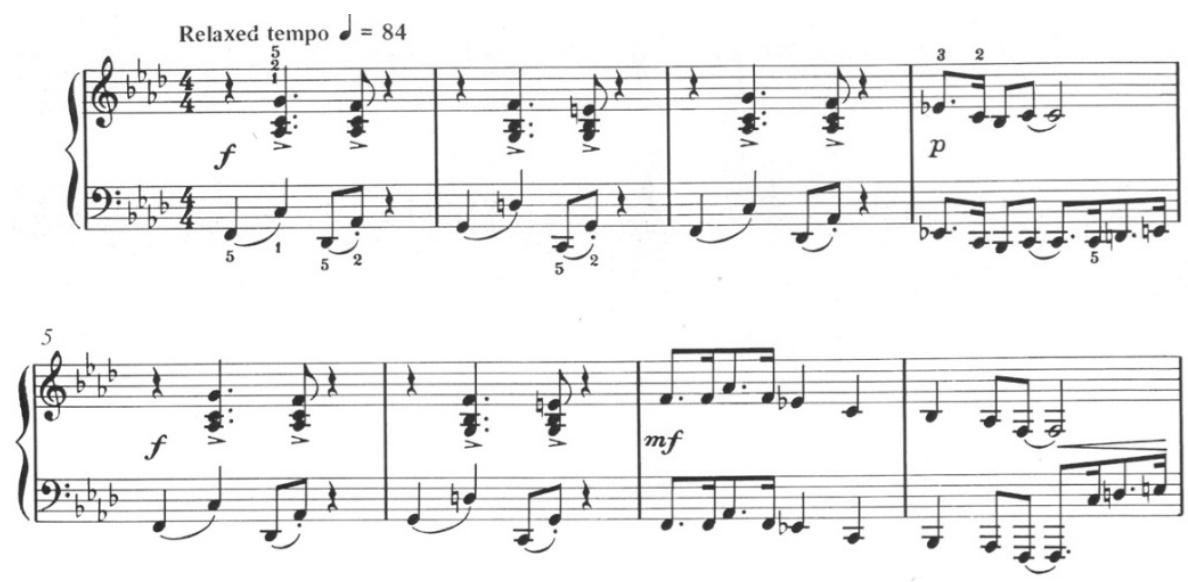

Fig. 7: Tema principal Laid-back blues

Las indicaciones de articulación son muy variadas y están muy relacionadas con la dirección del gesto (ligaduras) y el tipo de ataque (articulación).

Antes de ejecutar la pieza presta atención a estas indicaciones:

- Observa la armadura y la tonalidad de la pieza.

- Toca los acordes de I, IV, V, VI de la tonalidad principal.

- Practica la coordinación de los ritmos de la mano derecha e izquierda sobre la tapa del piano.

Ten en cuenta las siguientes cuestiones:

- ¿Cuántos acordes observas que hay por compás? Observa los compases 1 a 8.

- ¿Cuál crees que es el carácter de la pieza? ¿Cómo lo vas a conseguir? 